South African Journal of Geomatics, Vol. 7. No. 2, AfricaGEO 2018 Special Edition, September 2018

\title{
Investigating the impact of different types of directions on wayfinding efficiency in an informal settlement
}

\author{
Aphiwe Madubedube, Victoria Rautenbach, Serena Coetzee \\ Centre for Geoinformation Science, Department of Geography, Geoinformatics and \\ Meteorology, University of Pretoria, South Africa, $\underline{\text { 12328929@tuks.co.za }}$
}

DOI: http://dx.doi.org/10.4314/sajg.v7i2.5

\begin{abstract}
Informal settlements are a common occurrence in the South African landscape. These settlements are not planned and therefore lack conventional urban characteristics, such as street names and a regular grid or block structure, which are useful aids during wayfinding. As a result, it is difficult to navigate through such a settlement or to provide directions to a specific destination. The lack of street names also implies that directions provided by others play a more important role. In this paper, we present the results of a qualitative between-subjects study that assessed individuals' wayfinding efficiency when using different types of directions in an informal settlement. Participants were divided into two groups of six participants each ( 3 males and 3 females). Two sets of directions were constructed for the same route, one based on left-right turn descriptors and landmarks, and the second based on cardinal directions and distances. The performance of participants was measured for each set of directions. Earlier studies reported that people preferred left-right directions but performed better when using cardinal directions. The results of our study show the opposite. We think that this is due to the lack of regular wayfinding aids in informal settlements, but further studies are required to confirm this. The results of our study are interesting for developers of navigational tools aimed at addressing the needs of people in informal settlements.
\end{abstract}

\section{Introduction}

Wayfinding is defined as the mental process of finding a path between two points; a point of origin and a destination (Golledge, 1999; Xia, et al., 2008). Wayfinding (commonly referred to as navigating) often involves the use of instructions (i.e. directions) provided to assist with locating the destination. These instructions can be provided by an individual, a map or various other means (Nothegger et al., 2004; Hund \& Nazarczuk, 2009). Wayfinding is an integral part of everyday life, as we often have to rely on directions provided to us or provide directions to others (Hund \& Minarik, 2006; Hund \& Nazarczuk, 2009; Hund \& Padgitt, 2010; Hund \& Gill, 2014). For example, when visiting a new city, one may ask for directions to a local tourist attraction or well-known restaurant. These directions can be given using descriptive features, such as directions with 
reference to landmarks, street names, or cardinal directions and distances (Hund \& Minarik, 2006; Hund \& Padgitt, 2010; Hund \& Gill, 2014).

Hund and Padgitt (2010) stated that it is important to consider how familiar the individual providing and receiving the directions is with the environment, as the details provided might vary significantly. Based on individuals' opinions, directions that are based on landmarks and left-right descriptors are more common and found to be more understandable in an urban environment than cardinal directions (Hund et al 2008; Hund \& Padgitt, 2010). However, researchers have not yet been able to consistently find that one wayfinding strategies (e.g. using landmarks and street names compared to using cardinal directions and distances) for providing directions is better and more efficient than another (Hund \& Padgitt, 2010).

The type of wayfinding strategies followed often depends on the environment. Researchers have evaluated individuals' wayfinding efficiency in urban environments (i.e. virtual, physical, model towns and indoor) in American, Australian and European cities (Prestopnik \& Roskos-Ewoldsen, 2000; Lawton \& Kallai, 2002; Hund et al., 2008; Xia et al., 2008; Hund \& Padgitt, 2010; Padgitt \& Hund, 2012; Chang, 2013; Hund \& Gill, 2014; Walkowiak et al., 2015). These studies have focused on the descriptive features (i.e. information contained in different types of directions), gender differences and the influence of anxiety. The results of these studies have suggested that even though individuals prefer left-right directions, they often perform better when using cardinal directions in urban areas (Hund \& Minarik, 2006; Hund et al., 2008; Hund \& Padgitt 2010). Lawton and Kallai (2002) found that while men prefer directions based on a global reference point, women had a greater preference for route based information. However, all these studies were done using a structured urban environment and at the moment little is known about wayfinding in informal settlements. Informal settlements are a common occurrence in the South African landscape due to housing backlogs and the search for an employment in economic hubs (Rautenbach et al., 2015). These settlements are not guided by urban planning and thus lack the conventional elements, such as street names, typically used as wayfinding aids. As a result, it is difficult to navigate through such a settlement or provide directions to find/locate a specific destination. Not only is the lack of street names a challenge for wayfinding, but also the rapid changing and unstructured nature of informal settlements, and the terrain in some cases, for example steep slopes and rocky footpaths.

In this paper, we present results of a qualitative study that investigated individuals' wayfinding efficiency when using different types of directions in an informal settlement, considering that informal settlements are unstructured and without street signs. The remainder of the paper is structured as follows: in section 2, a brief background on the wayfinding, sense of direction and related work is provided; in section 3 the methodology is described; in section 4 the results are presented and discussed; and section 5 offers conclusions. 


\section{Background}

Farr et al., (2012) defines wayfinding as a process that involves finding your way to a destination within an environment (familiar or unfamiliar) through making use of the cues provided by the environment. Wayfinding has two components: movement and decision making (Xia et al., 2008). Movement refers to the mode of transport that one uses and to the duration to the destination. Decision making involves the wayfinding techniques and cognitive processes that people use while manoeuvring through an environment, and the decisions taken (i.e. selecting a specific path or route) to reach a destination. Golledge (1992) stated that most, if not all, our information about a specific location is gathered by travelling or moving through the environment, resulting in a more complete and detailed cognitive map of the environment. Thus, one can assume that an individual's familiarity with an environment plays an important role when deciding on a wayfinding strategy.

There are two main wayfinding strategies: orientation or survey strategies, and route strategies (Lawton, 1994; Golledge, 1999; Prestopnik \& Roskos-Ewoldsen, 2000; Hund \& Minarik, 2006; Hund \& Nazarczuk, 2009; Hund \& Padgitt, 2010; Chang, 2013; Hund \& Gill, 2014; Walkowiak et al., 2015). Orientation or survey strategies focus on keeping track of global reference points, for example, knowing where one is in relation to a certain reference point or global reference frame. Route strategies focus on routes and landmarks, for example, this strategy relies on directions to turn left at a particular landmark. Each of these strategies makes use of a different perspective of the environment. The survey strategy looks at the environment from an aerial perspective or birds eye view thus providing an overview of an environment (e.g. someone viewing a map), whereas the route strategy looks at the environment from the pedestrian perspective while moving through the environment (i.e. on a turn by turn basis) (Prestopnik \& Roskos-Ewoldsen, 2000; Hund et al., 2008; Hund \& Padgitt, 2010; Padgitt \& Hund, 2012; Chang, 2013; Hund \& Gill, 2014; Walkowiak et al., 2015). The selected strategy will influence performance, accuracy and efficiency while finding one's way through an environment (Hund \& Padgitt, 2010).

Individuals generally provide directions using left-right turns, cardinal directions or a mixture of both. These types of instructions have a direct link to the wayfinding strategies. Directions that rely on the route perspective include left-right turn descriptors and landmarks (e.g. turn right after the art gallery and your destination will be on the left). Survey directions tend to utilize reference frames, such as cardinal directions (i.e. north, east, south, west), the position of the sun, and distances, such as counting the number of city blocks or kilometres (Hund et al., 2008; Chang, 2013). Hund and Minarik (2006) conducted a user study where they provided participants with a set of directions to navigate through a model town. They found that participants who preferred survey strategies (i.e. cardinal directions) outperformed the participants who preferred route strategies (i.e. landmarks and left-right directions). In a later study, Hund and Padgitt (2010) again confirmed participants are more successful when using the cardinal descriptors. However, individuals prefer directions that contained more left-right descriptors, as opposed to cardinal descriptors, and feel that these directions would be more effective (Hund et al., 2008). 
The wayfinding strategy is only one factor to consider when investigating an individual's performance, accuracy and efficiency. Other factors, such as familiarity, gender, and sense of direction, can have an influence on how an individual would interpret the directions or provide the directions (Hund et al., 2008). Although sense of direction is not the main factor that could influence wayfinding, it often correlates with an individual's wayfinding abilities (Prestopnik \& Roskos-Ewoldsen, 2000; Hund \& Nazarczuk, 2009; Hund \& Padgitt, 2010).

\section{Methodology}

\subsection{Study design}

The aim of the qualitative study was to investigate how different types of directions influence wayfinding efficiency in an informal settlement. The experiment took place in Alaska, an informal settlement in Mamelodi East (see Figure 1). For the study, third year geoinformatics students of the University of Pretoria were recruited. A between-subjects design was used for the study, meaning that each participant was exposed to only one level of the independent variable. The participants were divided into two groups of six participants each ( 3 males and 3 females). Two sets of directions were constructed for the same route, one based on left-right turn descriptors and landmarks, and the second based on cardinal directions and distances.
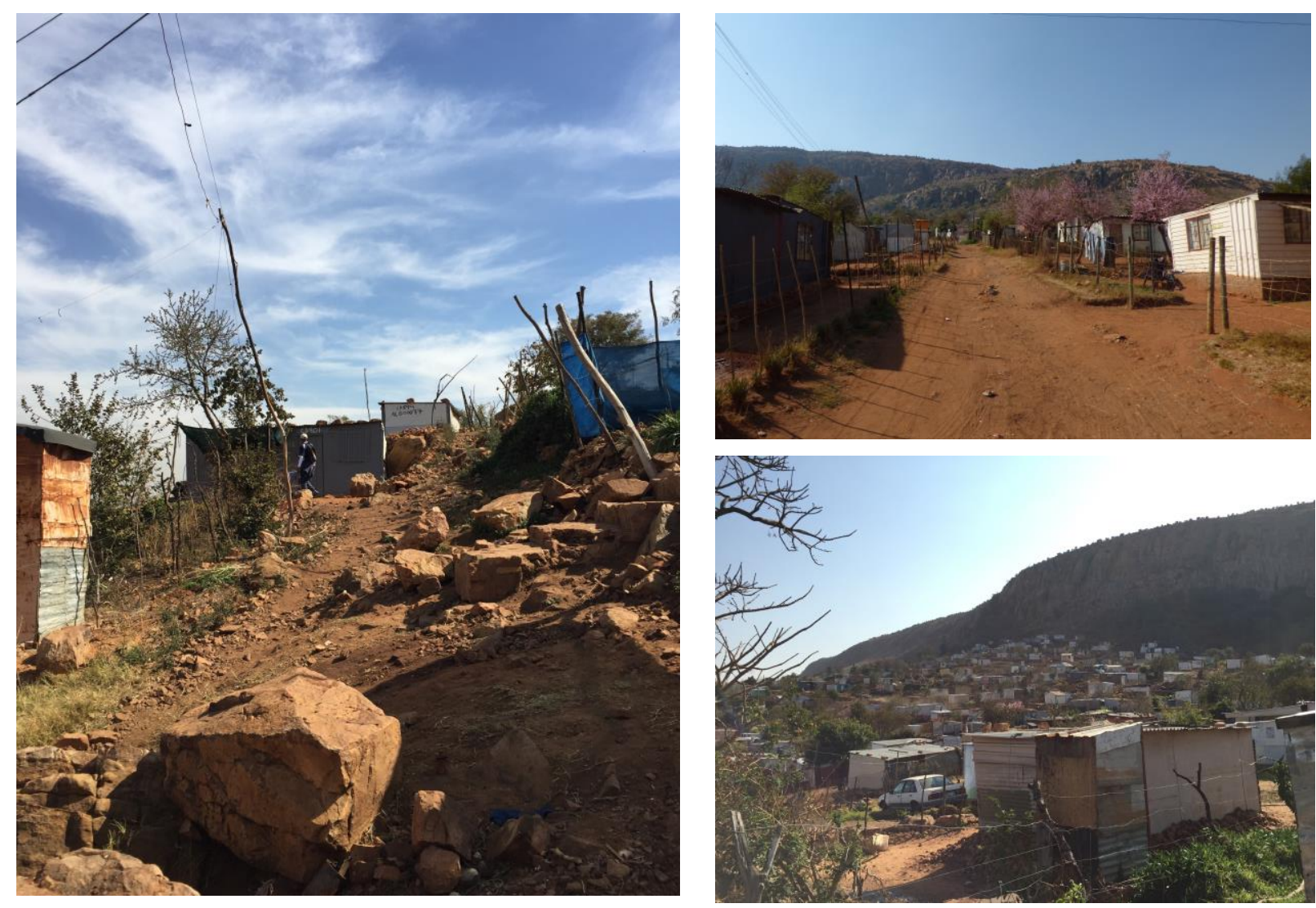

Figure 1. The rocky footpaths (left) and wide road (top right) in Alaska, Mamelodi that is situated at the foot of the Magaliesberg mountain (bottom right) 
The independent variable for this study was the directions, which comprised of either cardinal descriptors or left-right directions. The dependent variable was the performance of participants: accuracy (Did the participant manage to find the correct end location? Did the participant follow the correct route?), the number of times the participant stopped (e.g. to orientate themselves or to look down to refer to the directions), and any errors made. Based on (Hund et al., 2008; Hund \& Nazarczuk, 2009; Hund \& Padgitt, 2010), five actions considered to be errors: 1) going back to retrace one's steps (e.g. to fix any mistakes made); 2) making a wrong turn; 3) ending at an incorrect location; 4) missing the destination (e.g. moving past it or not reaching it); and 5) not completing the task or giving up. Time was not considered as a measure for efficiency as the terrain in the informal settlement is challenging (e.g. steep slopes, rocky footpaths and low hanging illegal electricity connections). We felt that if the time was recorded the participant might rush and this could possibly be a risk to their safety.

\subsection{Participants and materials}

Twelve geoinformatics students volunteered for the study. The participant age varied from 20 to 28 years with an average age of 22 . No compensation was given, and participation was completely voluntary. In the post-questionnaire, the participants indicated that 8 of them had been in an informal settlement prior to visiting Alaska, Mamelodi; only 4 participants had never visited an informal settlement. The participants also indicated that the majority (8) were not anxious about being in an informal settlement; 4 indicated that they were moderately anxious (based on a fivepoint Likert scale).

The directions were provided to the participants (refer to Table 1) and the participants were fitted with a GoPro Hero HD video camera before starting the study. No map or another information was provided to the participants.

Table 1: The two sets of directions provided for the wayfinding task (Task 2)

\section{Left-right directions}

From the Viva Foundation walk towards the circle (taxi stop).

Continue walking straight until you reach the footpath straight ahead.

Follow the footpath (curve with the footpath).

At the first intersection turn right and follow the footpath.

Take the first left turn and walk until you reach the dwelling painted in Brown.

At the brown dwelling turn left and follow the footpath.

At the end of the footpath turn right and walk up towards the mountain.

Take the first left turn and continue walking, you should be

\section{Cardinal directions}

From Viva Foundation head south, south east.

Continue heading south, south east and follow the footpath or 170 meters (curve with the footpath).

At the intersection head south east for 18 meters.

Turn and head north east for 118 meters.

Head north west until the end of the footpath (for 24 meters).

Head north east at the end of the footpath and walk for 39 meters.

At the intersection head north west for 14 meters.

Head south west for 94 meters.

Turn and head north west at the intersection and walk 
approaching a big rock.

At the big rock turn left and walk down until you reach the dwelling with a sugar cane garden.

After the dwelling turn right.

Walk towards the first intersection.

Turn left and walk down until the end of the footpath.

Walk towards the centre of the circle (taxi stop).

Turn right and walk towards the Viva foundation.

You have reached your destination. for 29 meters.

Head south west for 119 meters.

Head north, north west for 42 meters.

You have reached your destination.

\subsection{Procedure}

Participants were randomly divided into two groups (Group 1: left-right, Group 2: cardinal), as this is a between-subjects study. The study was conducted with one participant at a time. However, as the study was done within an informal settlement, the participants were not in a controlled environment and were requested not to speak to community members or fellow class mates (apart from a basic greeting) if they encountered them along the way. At the start of the study, each participant was informed of what to expect and any the instructions were explained, such as to limit external interaction.

The study consisted of three phases:

- For Task 1, each participant was asked to complete the Santa Barbara Sense of Direction (SBSOD) questionnaire prior to commencing with the wayfinding task. This questionnaire allows participants to rate their own sense of direction by answering a set of 15 questions related to wayfinding and sense of direction. Results from the SBSOD scale were used to aid in determining the role that sense of direction plays in wayfinding efficiency and to determine how accurately people's ratings are of what they perceive their own sense of direction to be.

- For Task 2, the participants completed the wayfinding task. The circular route that was used is shown in Figure 2. Prior to starting, participants were fitted with the GoPro head camera so that the entire experience could be recorded. This was done in order to gain insight into the processes followed by each participant while completing the wayfinding task. Participants in group 2 (i.e. cardinal directions) were asked to indicate North before starting the task. We recorded if the participant was correct or not. If a participant did not know where North was, or indicated it incorrectly, North was indicated to them before starting with the task. After being fitted with the head camera, participants were given directions that were either based on cardinal descriptors and distances or left-right descriptors and landmarks, depending on the group they had been assigned to (refer to Table 1). These instructions described how participants would get from a point of origin 
(point A) to a destination (point B) within the informal settlement. Aside from the information gathered using the camera, the instructor and a research assistant walked behind participants with a log book to observe and take notes of how well the task was performed and whether any errors or stops were made.

- For the final task (Task 3), participants were asked to complete a post-questionnaire to gather information regarding their feelings about informal settlements (i.e. if they had visited an informal settlement previously, and if they felt anxious about being in an informal settlement), and if the directions they had to follow were difficult.

\section{Results and discussion}

In this section, we first present the results of the Santa Barbara Sense of Direction (SBSOD) scale. Then we present the participants efficiency during the wayfinding task, focusing on the average number of errors per group (i.e. cardinal or left-right directions). Lastly, the participants performance based on their gender and anxiety is presented.

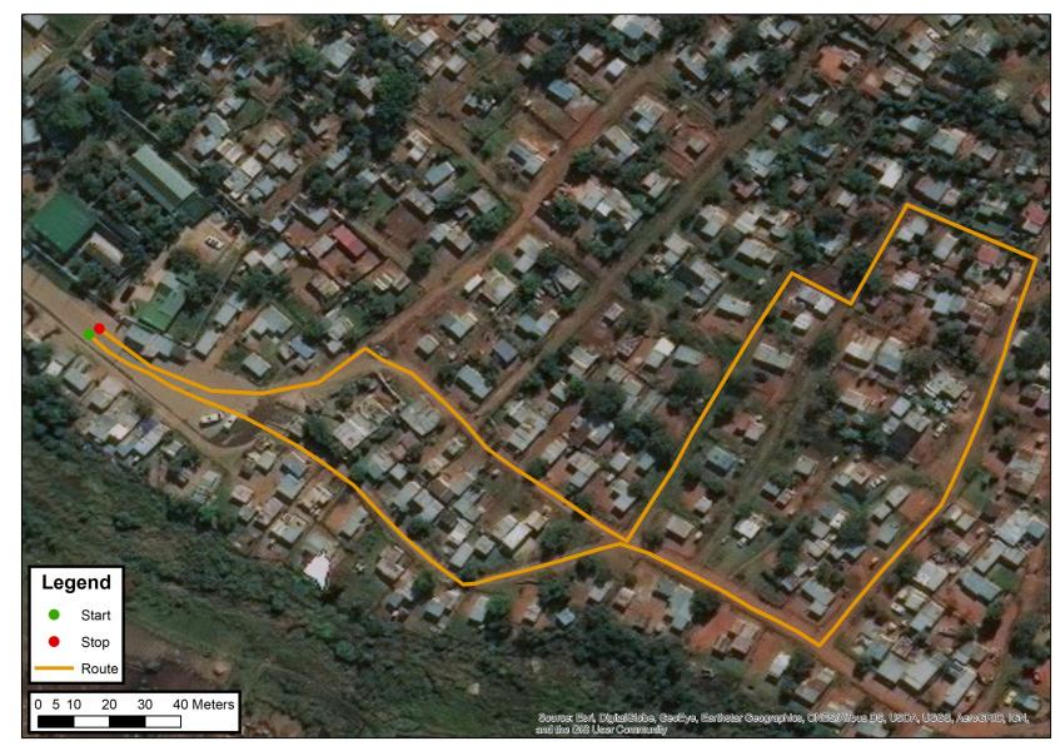

Figure 2. The route participants had to follow for the wayfinding task

\subsection{Participants self-reported sense of direction (SBSOD)}

The SBSOD scale is a self-reporting measure of an individual's spatial ability, specifically sense of direction. The participant's responses to each of the 15 Likert-scale questions is then combined to final score between 1-7, where a higher score indicates a better perceived sense of direction (Hegarty et al., 2002).

Figure 3 provides an overview of the results of the SBSOD scale, indicating each participant's gender, the group they were assigned to (i.e. cardinal or left-right directions), and the average value 
for each gender. No participant rated themselves extremely high, as the average of all participants was 4,80 (out of 7). The highest male score was 5,60 which was slightly higher than the highest female score of 5,13. In general, the male participants (average of 5,32) rated themselves higher than the female participants (average of 4,49). This confirms research which suggests that men have a better sense of direction than women (Bryant, 1982), however, they may also be overly confident. The participants who were assigned to the left-right directions group (average of 4,94) rated themselves slightly higher than the participants in the cardinal directions group (average of 4,66).

\subsection{Participant efficiency during the wayfinding task}

Figure 4 provides and overview of the number of errors per participant. The larger number of errors by participants in the cardinal directions group can be seen clearly. This is confirmed when the errors for the two groups (i.e. cardinal and left-right directions) are aggregated, as seen in Figure 5. The number of stops in the one group is almost double that in the other group, suggesting that the participants had to stop often to for example determine the cardinal direction required or to count the number of meters left. It is also interesting to note the participant with the lowest SBSOD score $(3,13)$ made 7 stops to verify the cardinal direction or estimate meters and only once did she retrace her steps. This is below the average number for the cardinal directions group as indicated in Figure 5 .

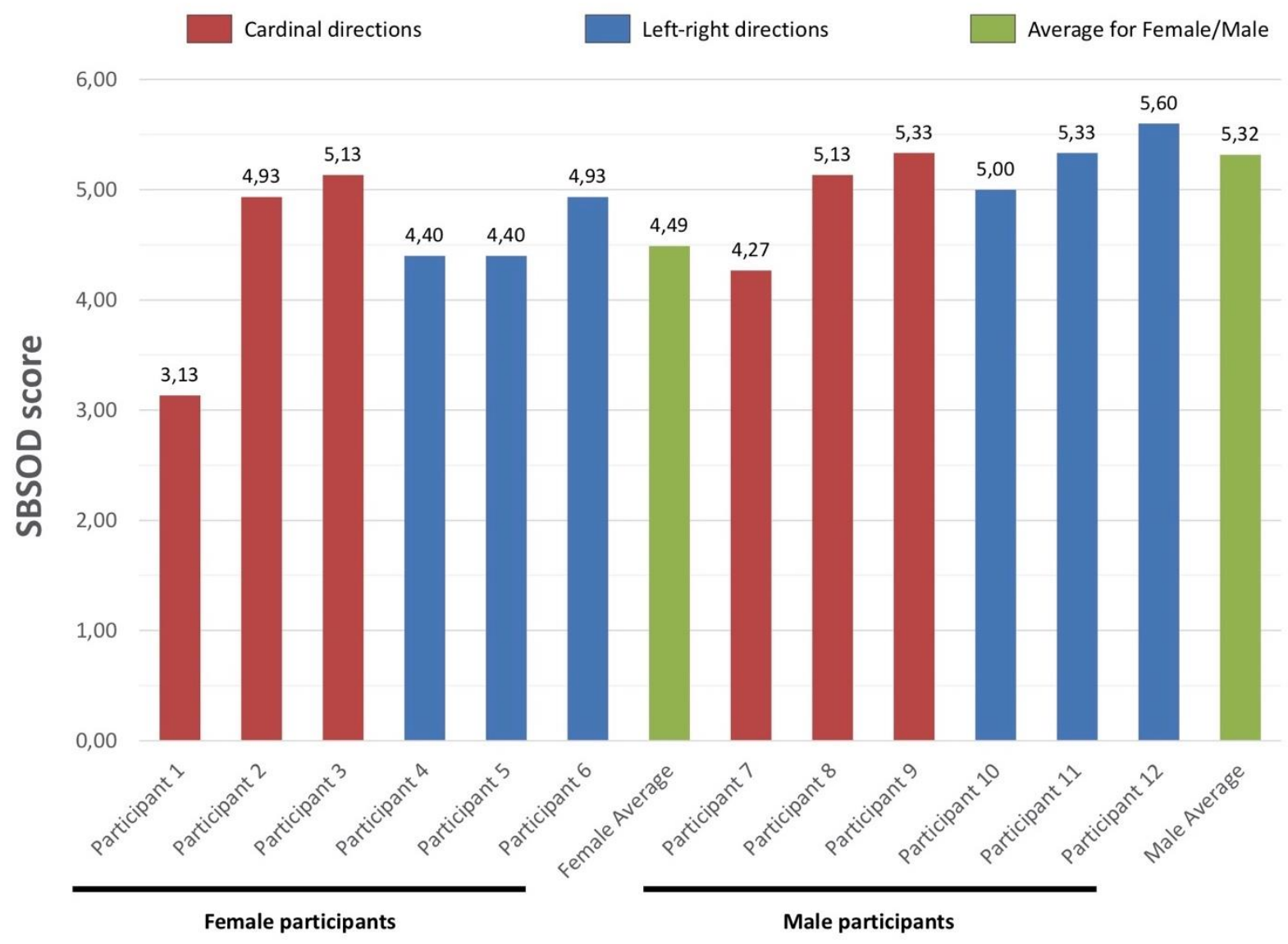


Figure 3. Participants' SBSOD scale scores

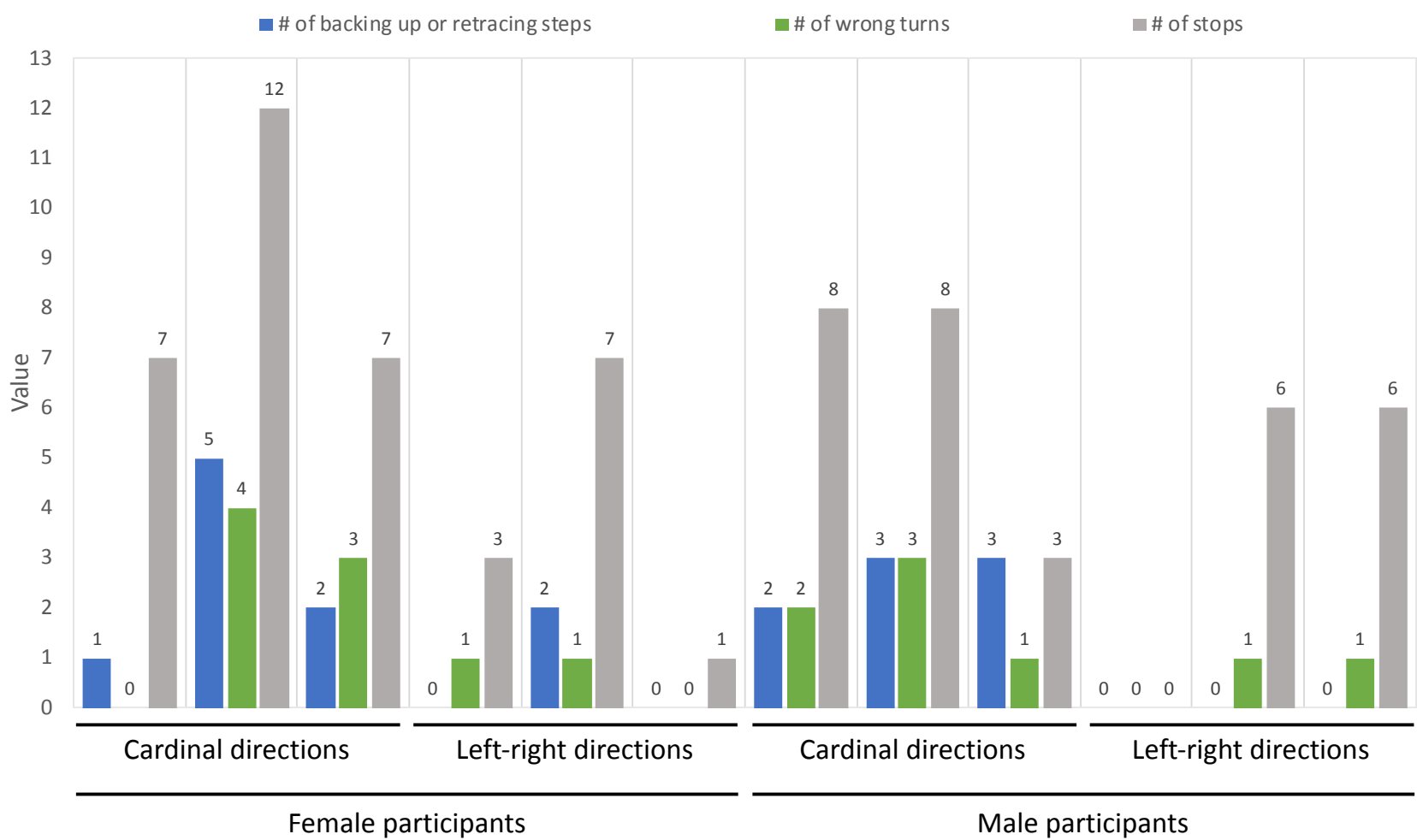

Figure 4. Number of wayfinding errors per participant

All participants, except one, were able to complete the wayfinding task without missing or moving past the destination. The participant who moved past the destination was the female participant with the highest SBSOD score and did not actually make many other errors during the task (i.e. 5 errors and 7 stops). This participant followed the cardinal directions where distances were given in meters. She was the tallest participant; and wrongly assumed that one of her steps was one meter.

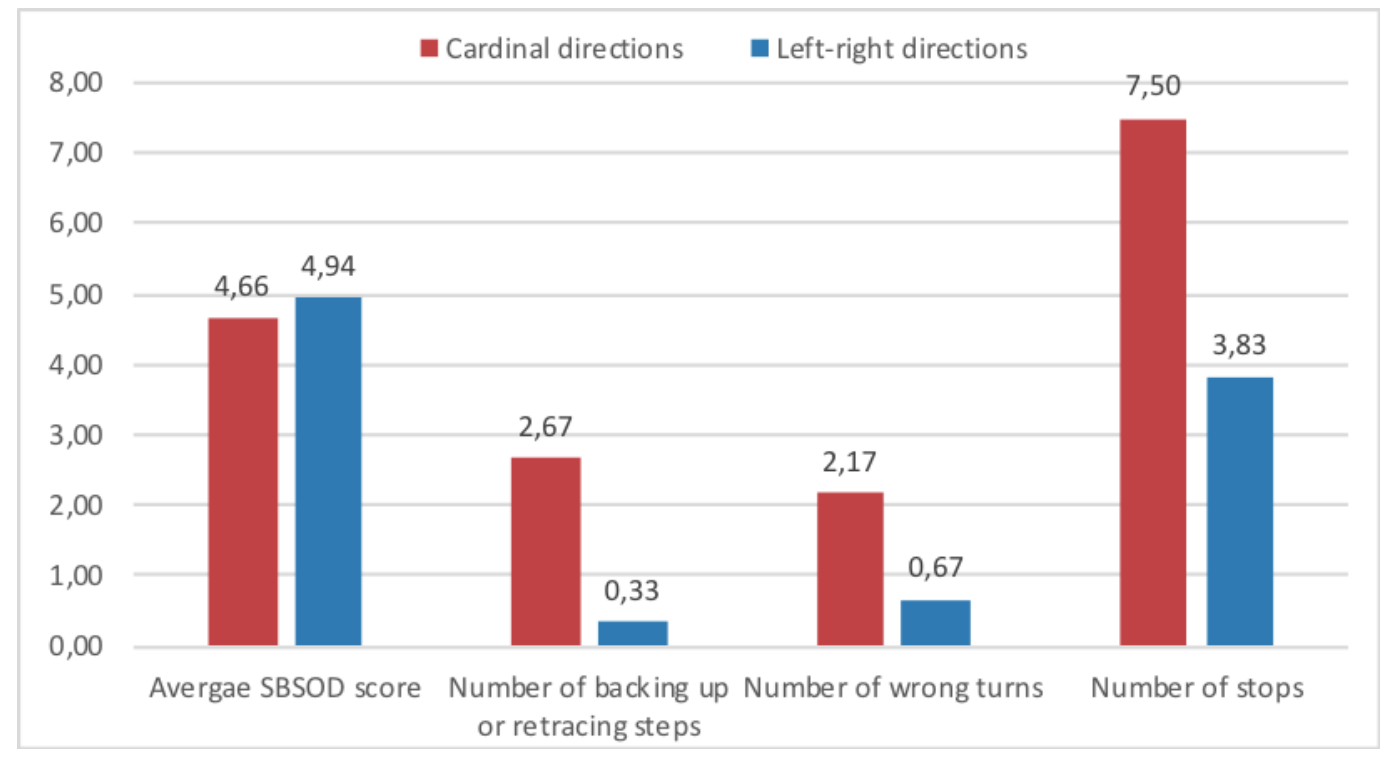


Figure 5. The average number of errors for each group

There was only one participant (male) who completed the task without any actions that could be considered to be errors. He was the participant who rated himself the highest on the SBSOD scale $(5,6)$. The participant was in the left-right group and was able to complete the task easily and with confidence.

\subsection{Group differences in performance based on gender and anxiety}

To better understand if the results vary based on participant characteristics, we studied the effect of gender. Literature suggests that males are better at wayfinding tasks than females (Bryant, 1982). Our results indicate the same trend, however, the differences are not significant. Refer to Figure 6.

Figure 7 and Figure 8 shows the average number of errors per group for both males and females. Although on average females made more errors than males, the difference is generally not more than 1 . The only exception would be that females made at least 2 more stops than males when using the cardinal directions. However, when using left-right directions on average the females made slightly fewer stops $(0,33)$ than their male counterparts.

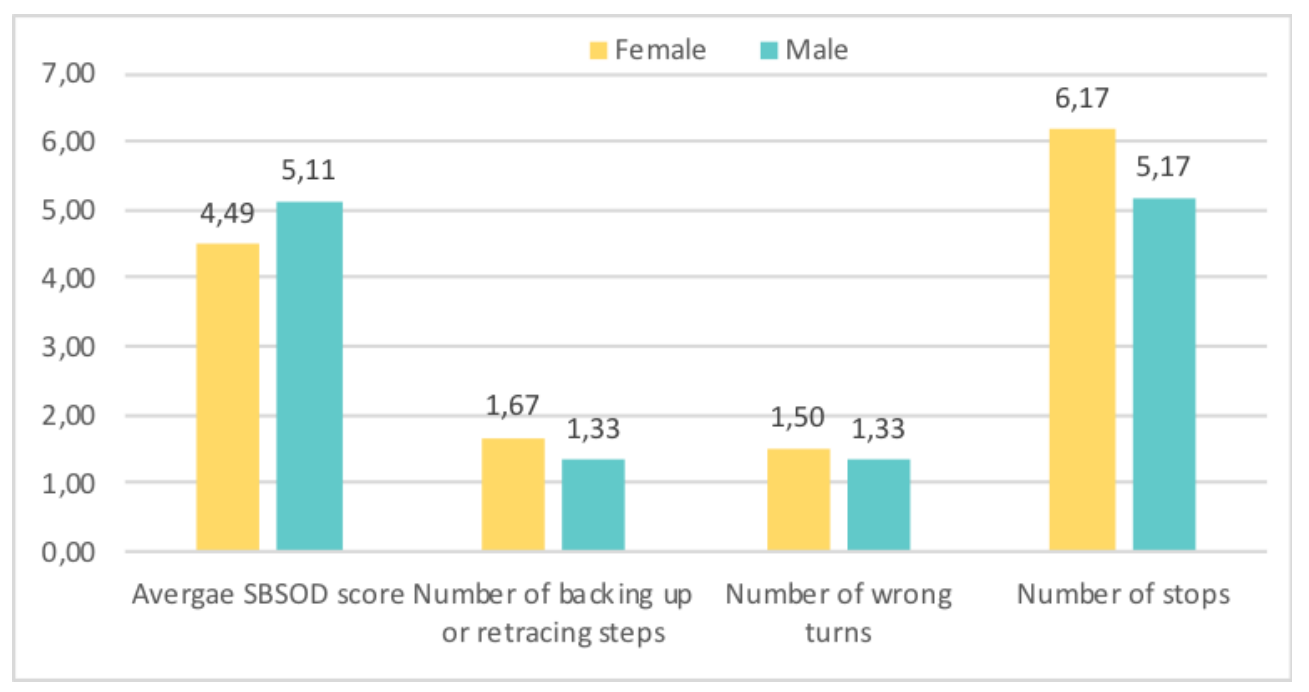

Figure 6. The average SBSOD score and number of errors of males and females 
South African Journal of Geomatics, Vol. 7. No. 2, AfricaGEO 2018 Special Edition, September 2018

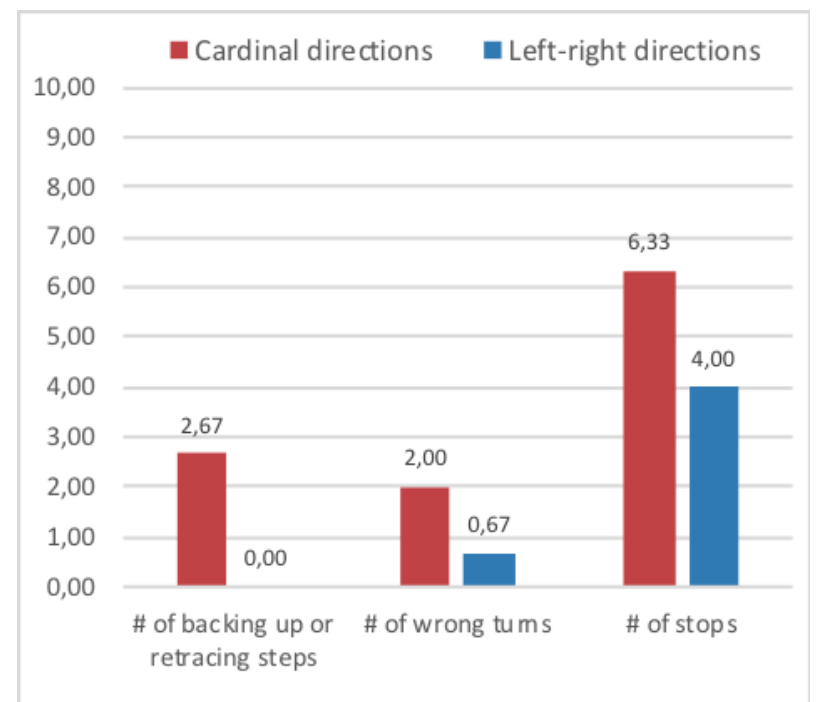

Figure 7. The average number of errors per group for males

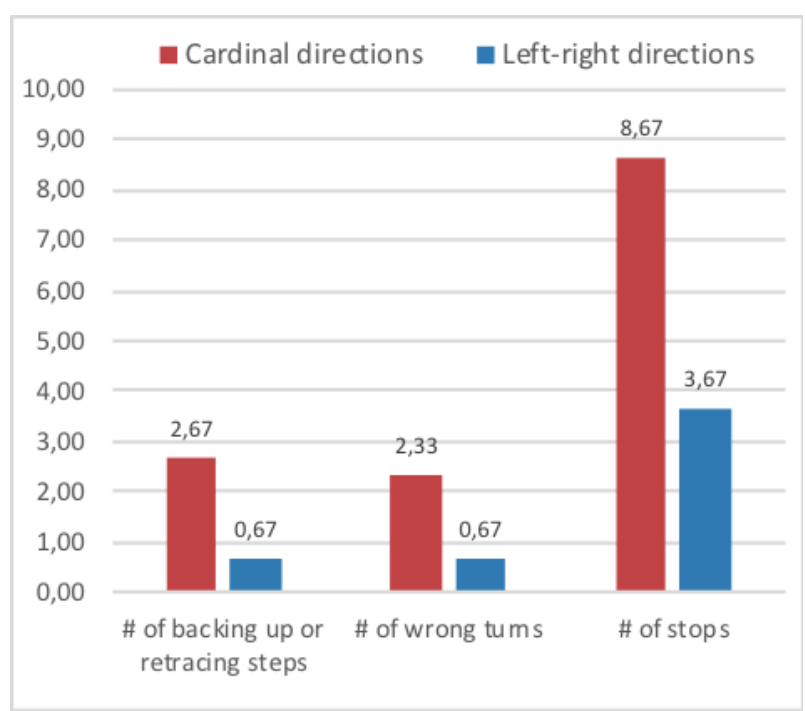

Figure 8 . The average number of errors per group for females

The participants who followed the cardinal directions reported higher levels of anxiety while completing the wayfinding task than the participant that used the left-right directions (refer to Figure 9). Lastly, the participants were asked to indicate how difficult the directions were to follow. See Figure 10. Overall, the participants perceived the cardinal directions to be more difficult to follow than the left-right directions. This correlated with the findings of Hund et al., (2008) that individuals prefer directions that are based on left-right descriptors and landmarks. However, unlike Hund and Padgitt (2010) the participants who followed the left-right directions were more efficient.

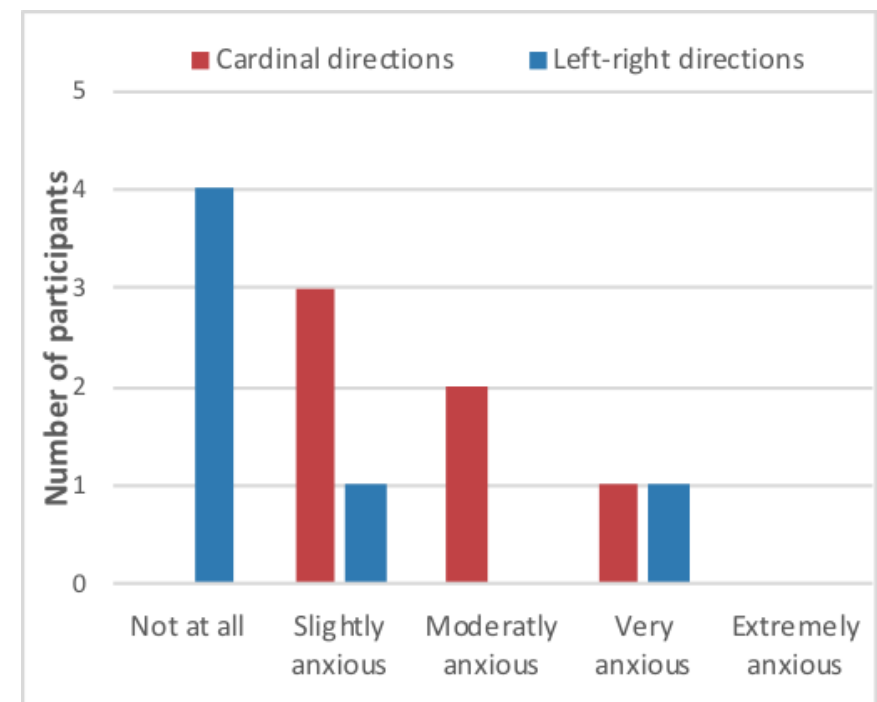

Figure 9. Participants self-reported level of anxiety while performing the wayfinding task per group

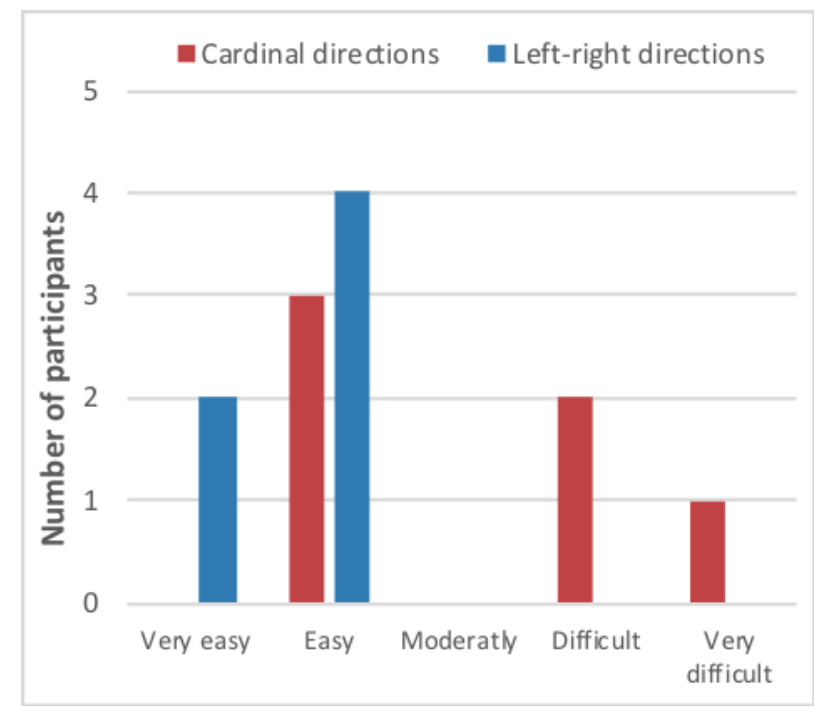

Figure 10. Participants perception of the difficulty of the directions they followed 


\section{Conclusion}

To summarise, the aim of the qualitative study presented in this article was to investigate how different types of directions influence wayfinding efficiency in an informal settlement. Little is known about wayfinding in informal settlements and how the rapidly changing and unstructured environment would affect an individual wayfinding efficiency. To investigate the wayfinding efficiency, we performed a between-subjects study where participants had to navigate a circular route through the informal settlement of Alaska in Mamelodi East.

Earlier studies in America, Australia and Europe reported that people preferred left-right directions but performed better when using cardinal directions (Hund \& Minarik, 2006; Hund et al., 2008; Hund \& Padgitt 2010). The results of our study show the opposite. We think that this is due to the lack of regular wayfinding aids in informal settlements and that in an informal settlement the routes might not always be as clear. For example, there is often two footpaths close to each other and an individual might not be able to distinguish them when estimating distance travelled. However, in an urban area this is clearer with city blocks, for example. However, further studies are required to confirm this. Similar to the previous studies (Hund \& Minarik, 2006; Hund et al., 2008; Hund \& Padgitt 2010), our participants also found the cardinal directions difficult to follow and preferred left-right directions.

The methods used in this study are qualitative, as the study was conducted under conditions that were not strictly controlled. While the informal settlement provided a real life situation, it also introduced some methodological challenges. The sample group used for this study has higher-thanaverage spatial abilities and the results may be different with the general population and even the community members. Additionally, various external influences could have affected the results, for example, the participants could have gained information about the wayfinding task from their fellow class mates or interpreted the instructor's actions while they were observing the task. However, it would not be possible to eliminate all these influences without conducting the study in a completely virtual environment. This would not be ideal as the study would lose the uniqueness of the environment as it cannot be simulated.

The results of our study are interesting for developers of navigational tools aimed at addressing the needs of people in informal settlements. Further research should investigate how navigational tools can be adapted for wayfinding in informal settlements.

\section{References}

Bryant, KJ 1982, 'Personality correlates of sense of direction and geographical orientation', Journal of Personality and Social Psychology, vol. 43, pp. 1318-1324.

Chang, H 2013, 'Wayfinding Strategies and Tourist Anxiety in Unfamiliar Destinations', Tourism Geographies, vol. 15, no. 3, pp. 529-550. 
Farr, AC, Kleinschmidt, T, Yarlagadda, P \& Mengersen, K 2012, 'Wayfinding: A simple concept, a complex process', Transport Reviews: A Transnational Transdisciplinary Journal, vol. 32, no. 6, pp. 715-743.

Golledge, RG 1992, 'Place Recognition and Wayfinding: Making Sense of Space', Geoforum, vol. 23, no. 2, pp. 199-214.

Golledge, RG 1999, Wayfinding Behaviour: Cognitive Mapping and Other Spatial Processes, Johns Hopkins University Press, Baltimore.

Hegarty, M, Richardson, AE, Montello, DR, Lovelace K \& Subbiah, I 2002, 'Development of a self-Report Measure of Environmental Spatial Ability', Intelligence, vol. 30, pp. 425-447.

Hund, AM \& Gill, DM 2014, 'What constitutes effective wayfinding directions: The interactive role of descriptive cues and memory demands', Journal of Environmental Psychology, vol. 38, pp. 217-224.

Hund, AM, Haney, KH \& Seanor, BD 2008, 'The role of recipient perspective in giving and following wayfinding directions', Applied Cognitive Psychology, vol. 22, pp. 896-916.

Hund, AM \& Minarik, JL 2006, 'Getting from Here to There: Spatial Anxiety, Wayfinding Strategies, Direction Type, and Wayfinding Efficiency', Spatial Cognition \& Computation, vol. 6, no. 3, pp. 179201.

Hund, AM \& Nazarczuk, SN 2009, 'The effects of sense of direction and training experience on wayfinding efficiency', Journal of Environmental Psychology, vol. 29, no. 1, pp. 151-159.

Hund, AM \& Padgitt, AJ 2010, 'Direction giving and following in the service of wayfinding in a complex indoor environment', Journal of Environmental Psychology, vol. 30, pp. 553-564.

Kozlowski, LT \& Bryant, KJ 1977, 'Sense of direction, spatial orientation, and cognitive maps', Journal of Experimental Psychology: Human Perception and Performance, vol. 3, pp. 590-598.

Lawton, CA 1994, 'Gender differences in wayfinding strategies: relationship to spatial ability and spatial anxiety', Sex Roles, vol, 30, pp. 765-779.

Nothegger, C, Winter, S \& Raubal, M 2004, 'Selection of Salient Features for Route Directions', Spatial Cognition \& Computation, vol. 4, no. 2, pp. 113-136.

Prestopnik, JL \& Roskos-Ewoldsen, B 2000, 'The Relations Among Wayfinding Strategy Use, Sense of Direction, Sex, Familiarity, and Wayfinding Ability', Journal of Environmental Psychology, vol. 20, pp. 177-191.

Rautenbach, V, Bevis, Y, Coetzee, S \& Combrinck, C 2015, 'Evaluating procedural modelling for 3D models of informal settlements in urban design activities', South African Journal of Science, vol. 111, no. $11 / 12$.

Walkowiak, S, Foulsham, T \& Eardley, AF 2015, 'Individual differences and personality correlates of navigational performance in the virtual route learning task', Computers in Human Behaviour, vol. 45, pp. 402-410.

Xia, J, Arrowsmith, C, Jackson, M \& Cartwright, W 2008, 'The Wayfinding Process Relationships Between Decision-making and Landmark Utility', Tourism Management, vol. 29, pp. 445-457. 